HETEROCYCLES 87 (7): 1553-1559 (2013)

DOI: $10.3987 / C O M-13-12735$

\title{
CYCLOPROPANATION OF CARBON-CARBON DOUBLE BONDS IN RING D OF ERGOT ALKALOIDS
}

\section{Mária Incze, , Gábor Dörnyei, ${ }^{a}$ Péter Kovács, ${ }^{a}$ Orsolya Egyed, ${ }^{b}$ György Hajós, ${ }^{a}$ and Csaba Szántay ${ }^{\mathrm{c}}$.}

${ }^{a}$ Department of Heterocyclic Chemistry, Institute of Organic Chemistry, Research Centre for Natural Sciences, Hungarian Academy of sciences, H-1525 Budapest, POB 17, Hungary, ${ }^{b}$ Department of NMR Spectroscopy, Institute of Organic Chemistry, Research Centre for Natural Sciences, Hungarian Academy of Sciences, H-1525 Budapest, POB 17, Hungary, ' Department of Organic Chemistry and Technology, University of Technology and Economics, H-1111 Budapest, Szt. Gellért tér 4., Hungary. E-mail: szantay@ttk.mta.hu

\begin{abstract}
Some ergot alkaloid derivatives containing a double bond in ring D have been reacted with diazomethane/palladium diacetate reagent to result in formation of a fused cyclopropane ring. This procedure proved to be generally applicable for cyclopropanation of Ergot alkaloids.
\end{abstract}

Some years ago thorough investigation has been carried out to achieve cyclopropanation of the double bond located in ring $\mathrm{D}$ of a $\mathrm{C}$-nor-ergoline derivative (1) in order to perform the total synthesis of the racemic form of cycloclavine (2) which is the only known alkaloid containing a fused cyclopropane ring. This goal has been reached by using diazomethane/diethyl ether reagent in the presence of palladium diacetate. ${ }^{1}$ To the best of our knowledge, this was the first example for formation of a fused cyclopropane ring in alkaloids.
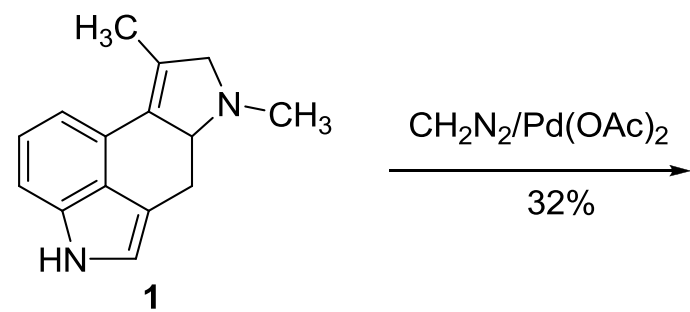<smiles>CN1CC2(C)CC23c2cccc4[nH]cc(c24)C[C@H]13</smiles> 
This successful transformation prompted us to extend our investigations in order to transform $\mathrm{C}=\mathrm{C}$ double bonds of various natural compounds to fused cyclopropane rings. Formation of the unique structural element of the cyclopropane moiety $y^{2,3}$ has always been considered as a challenge in the area of both artificial $^{4}$ and natural alkaloids. ${ }^{5-7}$ For such transformation various techniques (Simmons-Smith reaction ${ }^{8,9}$ transformation with dimethyloxosulfonium methylide, ${ }^{10}$ and reaction with diazomethane ${ }^{11}$ ) has been reported in the literature and, also, an extensive review have also been reported recently. ${ }^{12}$

In the course of our earlier research ${ }^{13,14}$ we described the synthesis of lysergic acid derivatives 3-5. These compounds containing the $\mathrm{C}-9=\mathrm{C}-10$ double bond seemed to serve as proper starting materials for the synthesis of new cyclopropane-fused derivatives. Thus, 3-5 were treated with diazomethane in the presence of palladium diacetate and, in accordance with our expectations, formation of the desired fused derivatives (6-8) was experienced. The compounds were isolated as hydrochloride salts in fairly poor yields (15-20\%) due to the high sensibility of the ergoline ring. Unfortunately other well known cyclopropanation methods ${ }^{8-11}$ failed as even traces of the desired compounds could not be detected in these cases.
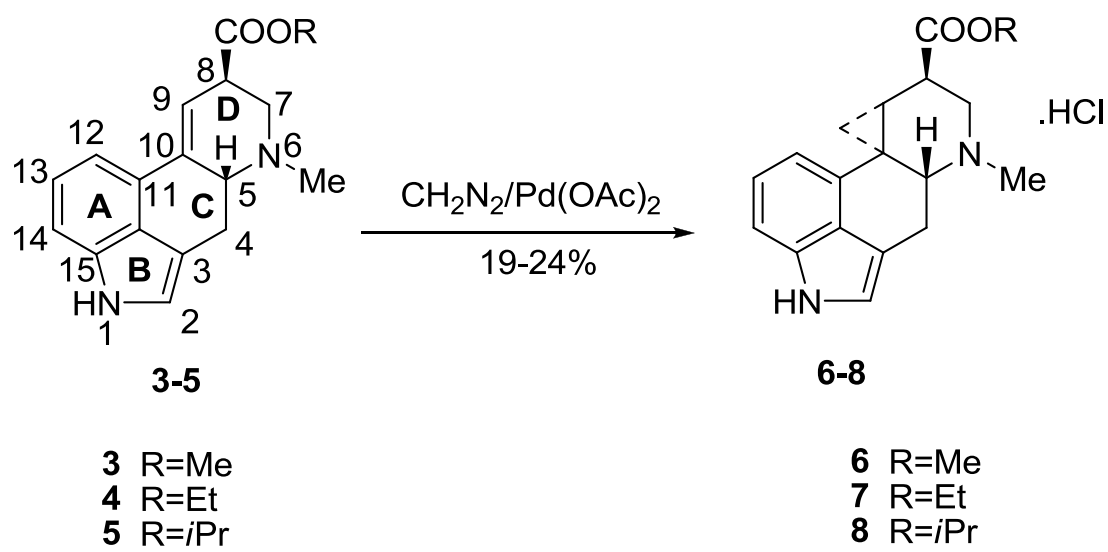

6-8

$6 \mathrm{R}=\mathrm{Me}$

$7 \mathrm{R}=\mathrm{Et}$

$8 \mathrm{R}=i \mathrm{Pr}$

Structures of the new compounds 6-8 have been elucidated by ${ }^{1}$ NMR spectroscopy. ${ }^{1}$ The steric arrangement of the cyclopropane ring was concluded upon results of NOE experiments: the new ring was found to occupy $\alpha$ position being in trans to H-5 atom.

In the synthetic pathway to lysergic acid an important intermediate is 8 -oxo-ergolene $(\mathbf{9})^{3}$ which was now also considered as a substrate for cyclopropanation. Treatment of $\mathbf{9}$ with diazomethane under the established reaction conditions gave a mixture of two products which were separated by chromatography. MS and ${ }^{1} \mathrm{H}-\mathrm{NMR}$ measurements unambiguously revealed that the cyclopropane ring was incorporated in both products (10 and 11). Spectral assignments suggested, furthermore, that the new cyclopropane ring 
had different steric positions in the two racemic compounds: the tricyclic ring occupied $\alpha$ position in the major product $\mathbf{1 0}$ isolated in $13 \%$ as a $\mathrm{HCl}$ salt, whereas it was found in $\beta$ position in the minor product $\mathbf{1 1}$ isolated in $6 \%$.
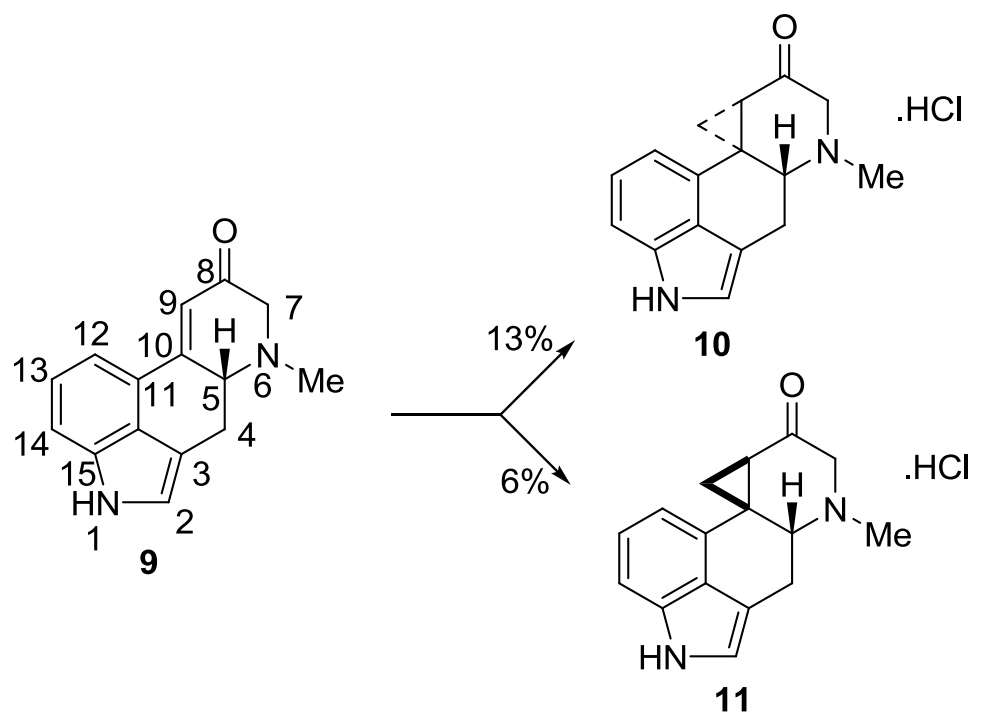

In order to rationalize preference of the $\alpha$ position of the cyclopropane rings formed in these reactions, heats of formation of the $\alpha$ and $\beta$ isomers were calculated by simple MM2 calculation. ${ }^{15}$ Comparison of the obtained values for heats of formation ( $37.67 \mathrm{Kcal}$ for $\mathbf{1 0}$ and 32.69 for $\mathbf{1 1}$ ) reveals that the $\alpha$ isomer $\mathbf{1 0}$ is more stable roughly by $5 \mathrm{kcal} / \mathrm{mol}$ in comparison to the $\beta$ isomer 11. Inspection of the 3D models of the two isomers suggest that in $\mathbf{1 1}$ the $\mathrm{CH}_{2}$ group of the cyclopropane ring and the $\mathrm{C} 12-\mathrm{H}$ atom are fairly close to each other. This steric hindrance as well as the possible 1,2-diaxial interaction between $\mathrm{CH}_{2}$ and the axial CHs might be responsible for the experienced stereoselectivity.

Successful cyclopropanation was also achieved with ergotamine (12) having a fairly complicated chemical structure. Thus, C-9 = C-10 double bond was found to undergo cyclopropanation to give the ring fused product $\mathbf{1 3}$, though the yield remained rather poor (12\%) in this case, too. 


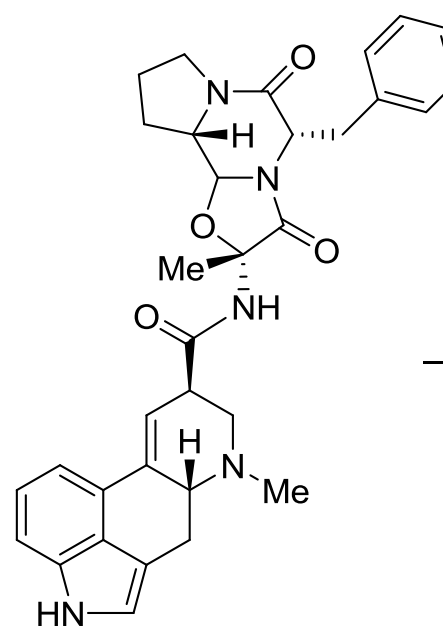

12

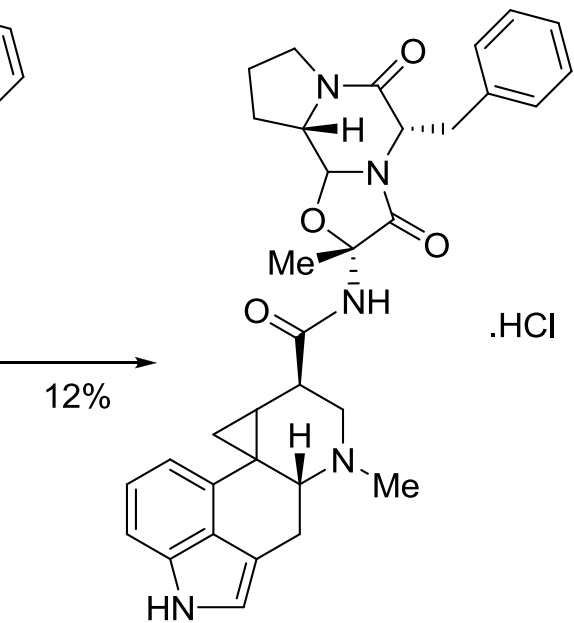

13

These findings reveal that our methodology for cyclopropanation seem to offer a general approach for transformation of the double bond of ring D of ergot alkaloids to a fused cyclopropane moiety. Further investigations in order to try to increase yields are in progress.

\section{EXPERIMENTAL}

\section{General methods}

Melting points are uncorrected. NMR measurements were performed on Varian INOVA-400 spectrometer equipped with a $5 \mathrm{~mm}$ inverse detection z-gradient probe. ${ }^{1} \mathrm{H}$ and ${ }^{13} \mathrm{C}$ NMR spectra were measured at $\mathrm{rt}$ $\left(25^{\circ} \mathrm{C}\right)$ using DMSO-d6 as solvent. ${ }^{1} \mathrm{H}$ and ${ }^{13} \mathrm{C}$ chemical shifts are referenced to residual solvent signals. ${ }^{1} \mathrm{H}$ and ${ }^{13} \mathrm{C}$ NMR spectra were acquired with standard conditions. The complete signal assignment was performed by running homo- (2D-NOESY) and hetero-correlation $\left({ }^{1} \mathrm{H}_{-}{ }^{13} \mathrm{C}\right.$ gHSQC and ${ }^{1} \mathrm{H}-{ }^{13} \mathrm{C}$ gHMBC) measurements. The pulse programs were taken from the Varian software library. 2D NOESY experiments were acquired with a mixing time of $500 \mathrm{~ms}$, a recycle delay of $2 \mathrm{~s}$. Preparative separations were performed by column chromatography on Merck Kieselgel 60 (0.063-0.200).

\section{General procedure for cyclopropanation}

Solution of starting compounds $3,4,5,9$ or $12(1 \mathrm{mmol})$ in $\mathrm{CH}_{2} \mathrm{Cl}_{2}(10 \mathrm{~mL})$ was cooled in an ice-water bath, $\mathrm{Pd}(\mathrm{OAc})_{2}(15 \mathrm{mg} ; 0.068 \mathrm{mmol})$ was added, and while stirred in an argon atmosphere, $0.3 \mathrm{M}$ $\mathrm{CH}_{2} \mathrm{~N}_{2}-\mathrm{CH}_{2} \mathrm{Cl}_{2}$ solution (35 mL; $10 \mathrm{mmol}$ ) was added into the mixture. The mixture was stirred at $0-5^{\circ} \mathrm{C}$. Progress of the reaction was checked by TLC $\left(\mathrm{CHCl}_{3}-\mathrm{MeOH}\right.$ 9:1 $\mathrm{R}_{f}$ of product $>\mathrm{R}_{f}$ of starting compound). After $1 \mathrm{~h}$ reaction time another portion of $\mathrm{CH}_{2} \mathrm{~N}_{2}-\mathrm{CH}_{2} \mathrm{Cl}_{2}$ solution $(35 \mathrm{~mL} ; 10 \mathrm{mmol})$ and $\mathrm{Pd}(\mathrm{OAc})_{2}(15 \mathrm{mg}$; $0.068 \mathrm{mmol}$ ) was added, and addition of the same amount of the reagent was repeated again after another 3 h. The conversion was then found to be stopped, the mixture was filtered, the solution dried over $\mathrm{MgSO}_{4}$ 
and evaporated. The residue was purified by column chromatography. The crude product was isolated as a $\mathrm{HCl}$ salt $\left(\mathrm{CH}_{2} \mathrm{Cl}_{2}-\mathrm{Et}_{2} \mathrm{O}+\mathrm{HCl} /\right.$ dioxane $)$.

\section{Methyl (9ßH)-6-methylcyclopropa[9,10]ergoline-8ß-carboxylate hydrochloride (6)}

Yield: $21 \%$, Mp: $165-170{ }^{\circ} \mathrm{C},[\alpha]_{D}^{25}+20.1(\mathrm{c} 0.5, \mathrm{MeOH})$

${ }^{1} \mathrm{H}$ NMR (400 MHz, DMSO-d6) $\delta$ (ppm): 0.64 (1H, dd $\left.J=9.5,5.2 \mathrm{~Hz}, \mathrm{H} 9 \mathrm{y}\right), 1.88\left(1 \mathrm{H}, \mathrm{t}, J=5.2 \mathrm{~Hz}, \mathrm{H} 9_{\mathrm{x}}\right.$ ), 2,52 (1H, ddd, $J=9.5,5.2,2.1 \mathrm{~Hz}, \mathrm{H} 9), 2.88(1 \mathrm{H}, \mathrm{t}, J=13.8 \mathrm{~Hz}, \mathrm{H} 4 \mathrm{y}), 2.92\left(3 \mathrm{H}, \mathrm{s}, \mathrm{N}^{+}-\mathrm{CH}_{3}\right), 3.21(1 \mathrm{H}, \mathrm{m}$, H7y), 3. 41 (1H, m, H2), $3.52\left(1 \mathrm{H}, \mathrm{m}, \mathrm{H} 7_{\mathrm{x}}\right), 3.61\left(1 \mathrm{H}, \mathrm{dd}, J=13.8,3.2 \mathrm{~Hz}, \mathrm{H} 4_{\mathrm{x}}\right), 3.86(1 \mathrm{H}, \mathrm{m}, \mathrm{H} 5), 6.51$ $(1 \mathrm{H}, \mathrm{d}, J=7.3 \mathrm{~Hz}, \mathrm{H} 12), 6.98(1 \mathrm{H}, \mathrm{dd}, J=8.1,7.3 \mathrm{~Hz}, \mathrm{H} 13), 7.10(1 \mathrm{H}, \mathrm{s}, \mathrm{H} 2), 7.14(1 \mathrm{H}, \mathrm{d}, J=8.1 \mathrm{~Hz}, \mathrm{H} 14)$, 10.9 (1H, s, H1), 10.95 (1H, br.s, $\left.\mathrm{N}^{+}-\mathrm{H}\right)$.

${ }^{13} \mathrm{C}$ NMR (100 MHz, DMSO-d6) $\delta$ (ppm): 14.5 (C9a), 20.4 (9), 24.2 (C4), 24.8 (C11), 37.5 (C8), 41.9 $\left(\mathrm{N}-\underline{\mathrm{CH}}_{3}\right), 52.2\left(\mathrm{O}-\underline{\mathrm{CH}}_{3}\right), 53.5(\mathrm{C} 7), 63.3$ (C5), 107.1 (C3), 108.5 (C12), 109.2 (C14), 119.1 (C2), 122.1 (C13), 125.6 (C15b), 131.8 (C12), 132.8 (C15a), 170.9 (ㅁO-O-CH $)_{3}$.

Anal. Calcd. for $\mathrm{C}_{18} \mathrm{H}_{21} \mathrm{ClN}_{2} \mathrm{O}_{2}(332,82) \mathrm{C}, 64.96 ; \mathrm{H}, 6.36 ; \mathrm{N}, 8.42$. Found, C, 64.70; H, 6.19; N, 8.19.

\section{Ethyl (9ßH)-6-methylcyclopropa[9,10]ergoline-8ß-carboxylate hydrochloride (7)}

Yield: $24 \%, \mathrm{Mp}: 146-156^{\circ} \mathrm{C},[\alpha]_{D}^{25}+15.8(\mathrm{c} 0.5, \mathrm{MeOH})$

${ }^{1} \mathrm{H}$ NMR (400 MHz, DMSO-d6) $\delta$ (ppm): $0.64(1 \mathrm{H}, \mathrm{dd} J=9.4,5.2 \mathrm{~Hz}, \mathrm{H} 9 \mathrm{y}), 1.25(3 \mathrm{H}, \mathrm{t}, J=7.0 \mathrm{~Hz}$, $\left.\mathrm{CO}-\mathrm{O}-\mathrm{CH}_{2}-\underline{\mathrm{C}}_{3}\right), 1.90(1 \mathrm{H}, \mathrm{t}, J=5.2 \mathrm{~Hz}, \mathrm{H} 9 \mathrm{x}), 2.50(1 \mathrm{H}, \mathrm{ddd}, J=9.4,5.2,2.0 \mathrm{~Hz}, \mathrm{H} 9), 2.88(1 \mathrm{H}, \mathrm{t}, J=12.9$ $\mathrm{Hz}, \mathrm{H} 4 \mathrm{y}), 2.92\left(3 \mathrm{H}, \mathrm{s}, \mathrm{N}^{+}-\mathrm{CH}_{3}\right), 3.20(1 \mathrm{H}, \mathrm{t}, J=11.5 \mathrm{~Hz}, \mathrm{H} 7 \mathrm{y}), 3.40(1 \mathrm{H}, \mathrm{m}, \mathrm{H} 2), 3.51(1 \mathrm{H}, \mathrm{dd}, J=11.5,6.1$ $\mathrm{Hz}, \mathrm{H} 7 \mathrm{x}), 3.61(1 \mathrm{H}, \mathrm{dd}, J=12.9,3.2 \mathrm{~Hz}, \mathrm{H} 4$ x $), 3.84(1 \mathrm{H}, \mathrm{m}, \mathrm{H} 5), 4.19\left(2 \mathrm{H}, \mathrm{q}, J=7.0 \mathrm{~Hz}, \mathrm{CO}-\mathrm{O}-\mathrm{C}_{2}-\mathrm{CH}_{3}\right)$, $6.61(1 \mathrm{H}, \mathrm{d}, J=7.4 \mathrm{~Hz}, \mathrm{H} 12), 6.98(1 \mathrm{H}, \mathrm{dd}, J=7.9,7.4 \mathrm{~Hz}, \mathrm{H} 13), 7.10(1 \mathrm{H}, \mathrm{s}, \mathrm{H} 2), 7.14(1 \mathrm{H}, \mathrm{d}, J=7.9 \mathrm{~Hz}$, H14), 10.9 (1H, s, H1), 11.0 (1H, br.s N $\left.{ }^{+}-\mathrm{H}\right)$.

${ }^{13} \mathrm{C}$ NMR (100 MHz, DMSO-d6) $\delta$ (ppm): $14.0\left(\mathrm{CO}-\mathrm{O}-\mathrm{CH}_{2}-\underline{\mathrm{CH}}_{3}\right), 14.8$ (C9a), 20.4 (C9), 24.2 (C4), 25.0 (C10), $37.7(\mathrm{C} 2), 41.7\left(\mathrm{~N}^{+}-\underline{\mathrm{C}} \mathrm{H}_{3}\right), 53.1$ (C7), $61.3\left(\mathrm{CO}-\mathrm{O}-\underline{\mathrm{CH}}_{2}-\mathrm{CH}_{3}\right), 62.9$ (C5), 107.7 (C4a), 108.9 (C12), 109.4 (C14), 119.1 (C2), 122.3 (C13), 126.2 (C15), 132.7 (C11), 133.0 (C15a), 170.9 (ㅁ-O- $\mathrm{CH}_{2}-\mathrm{CH}_{3}$ ),. Anal. Calcd. for $\mathrm{C}_{19} \mathrm{H}_{23} \mathrm{ClN}_{2} \mathrm{O}_{2}$ (346,85): C, 65.79; H, 6.68; N, 8.08. Found, C, 65.52; H, 6.39; N, 7.85.

\section{Isopropyl (9ßH)-6-methylcyclopropa[9,10]ergoline-8ß-carboxylate hydrochloride (8)}

Yield: $19 \%$, Mp: $155-160{ }^{\circ} \mathrm{C},[\alpha]^{25}+9.0$ (c 1.2, EtOH)

${ }^{1} \mathrm{H}$ NMR (400 MHz, DMSO-d6) $\delta$ (ppm): $0.67(1 \mathrm{H}, \mathrm{dd}, J=9.7,5.3 \mathrm{~Hz}, \mathrm{H} 9 \mathrm{y}), 1.26$ and $1.29(2 \times 3 \mathrm{H}, \mathrm{d}, J=$ $\left.6.3 \mathrm{~Hz}, \mathrm{CO}-\mathrm{O}-\mathrm{CH}-\left(\mathrm{CH}_{3}\right)_{2}\right)$ ), $1.97\left(1 \mathrm{H}, \mathrm{t}, J=5.3 \mathrm{~Hz}, \mathrm{H} 9_{\mathrm{x}}\right), 2.50(1 \mathrm{H}, \mathrm{m}, \mathrm{H} 9), 2.93\left(1 \mathrm{H}, \mathrm{m}, \mathrm{H} 4_{\mathrm{y}}\right), 2.94(3 \mathrm{H}$, s, $\left.\mathrm{N}^{+}-\mathrm{CH}_{3}\right), 3.22(1 \mathrm{H}, \mathrm{m}, \mathrm{H} 7 \mathrm{y}), 3.40(1 \mathrm{H}, \mathrm{ddd}, J=12.1,6.2,1.7 \mathrm{~Hz}, \mathrm{H} 8), 3.52(1 \mathrm{H}, \mathrm{dd}, J=12.4,6.3 \mathrm{~Hz}$, 
$\left.\mathrm{H} 7_{\mathrm{x}}\right), 3.64\left(1 \mathrm{H}, \mathrm{dd}, J=14.2,3.5 \mathrm{~Hz}, \mathrm{H} 4_{\mathrm{x}}\right), 3.88(1 \mathrm{H}, \mathrm{m}, \mathrm{H} 5), 5.03\left(1 \mathrm{H}, \mathrm{m}, \mathrm{CO}-\mathrm{O}-\mathrm{C} \underline{\mathrm{H}}-\left(\mathrm{CH}_{3}\right)_{2}\right), 6.53(1 \mathrm{H}, \mathrm{d}$, $J=7.2 \mathrm{~Hz}, \mathrm{H} 12), 7.01(1 \mathrm{H}, \mathrm{dd}, J=7.8,7.2 \mathrm{~Hz}, \mathrm{H} 13), 7.13(1 \mathrm{H}, \mathrm{s}, \mathrm{H} 2), 7.17$ (1H, d, $J=7.8 \mathrm{~Hz}, \mathrm{H} 14), 10.9$ $(1 \mathrm{H}, \mathrm{s}, \mathrm{H} 1), 11.1\left(1 \mathrm{H}\right.$, br.s $\left.\mathrm{N}^{+}-\mathrm{H}\right)$.

${ }^{13} \mathrm{C}$ NMR (100 MHz, DMSO-d6) $\delta$ (ppm): 14.8 (C9a), 20.3 (C9), $21.4+21.5$ (CO-O-CH-( CH$\left.\left._{3}\right)_{2}\right), 24.1$ (C4), 24.9 (C10), $37.8(\mathrm{C} 8), 41.7\left(\mathrm{~N}^{+}-\underline{\mathrm{CH}}_{3}\right), 53.1$ (C7), 62.9 (C5), 68.9 (CO-O- $\left.\underline{\mathrm{CH}}-\left(\mathrm{CH}_{3}\right)_{2}\right), 107.7(\mathrm{C} 4)$, 108.8 (C2), 109.4 (C14), 119.7 (C2), 122.3 (C13), 126.2 (C15b), 132.6 (C11), 133.0 (C15a), 170.5 ( $\left.\underline{\mathrm{CO}}-\mathrm{O}-\mathrm{CH}-\left(\mathrm{CH}_{3}\right)_{2}\right)$.

Anal. Calcd. for $\mathrm{C}_{20} \mathrm{H}_{25} \mathrm{ClN}_{2} \mathrm{O}_{2}(360,88)$ : C, 66.56; H, 6.98; N, 7.76. Found, C, 66.29; H, 6.89; N, 7.52.

\section{(9ßH)-6-Methylcyclopropa[9,10]ergoline-8-one hydrochloride (10)}

Yield: $13 \%, \mathrm{Mp}: 166-170{ }^{\circ} \mathrm{C},[\alpha]_{D}^{25}+21.5(\mathrm{c} 1.2, \mathrm{EtOH})$

${ }^{1} \mathrm{H}$ NMR (400 MHz, DMSO-d6) $\delta$ (ppm): 2.01 (1H, dd, $\left.J=10.4,5.4 \mathrm{~Hz}, \mathrm{H} 9\right), 2.43$ (1H, dd $J=10.3,6.9 \mathrm{~Hz}$, H9y $), 2.88\left(3 \mathrm{H}, \mathrm{s}, \mathrm{N}^{+}-\mathrm{CH}_{3}\right), 3.00(1 \mathrm{H}, \mathrm{dd}, J=14.0,12.8 \mathrm{~Hz}, \mathrm{H} 4 \mathrm{y}), 3.24\left(1 \mathrm{H}, \mathrm{m}, \mathrm{H} 9_{\mathrm{x}}\right), 3.44(1 \mathrm{H}, \mathrm{dd}, \mathrm{J}=14.0$, $\left.4.3 \mathrm{~Hz}, \mathrm{H} 4_{\mathrm{x}}\right), 3.89\left(2 \mathrm{H}, \mathrm{m}, \mathrm{H} 7_{\mathrm{x}}+\mathrm{H} 7_{\mathrm{y}}\right), 4.40(1 \mathrm{H}, \mathrm{dd}, \mathrm{J}=12.8,4.3 \mathrm{~Hz}, \mathrm{H} 5), 6.72(1 \mathrm{H}, \mathrm{d}, J=7.2 \mathrm{~Hz}, \mathrm{H} 12)$, $7.06(1 \mathrm{H}, \mathrm{dd}, J=7.8,7.2 \mathrm{~Hz}, \mathrm{H} 13), 7.18(1 \mathrm{H}, \mathrm{s}, \mathrm{H} 2), 7.22(1 \mathrm{H}, \mathrm{d}, J=7.8 \mathrm{~Hz}, \mathrm{H} 14), 11.0(1 \mathrm{H}, \mathrm{s}, \mathrm{H} 1), 11.5$ $\left(1 \mathrm{H}\right.$, br.s $\left.\mathrm{N}^{+}-\mathrm{H}\right)$.

${ }^{13} \mathrm{C}$ NMR (100 MHz, DMSO-d6) $\delta$ (ppm): 14.2 (C9), 20.0 (C4), 30.3 (C10), 36.2 (C9a), $41.2\left(\mathrm{~N}^{+}-\mathrm{CH}_{3}\right)$, 53.9 (C7), 57.4 (C5), 106.2 (C4), 110.0 (C14), 110.5 (C12), 120.3 (C2), 122.4 (C13), 126.0 (C15b), 128.1 (C11), 133.2 (C15), 196.8 (C8).

Anal. Calcd. for $\mathrm{C}_{16} \mathrm{H}_{17} \mathrm{ClN}_{2} \mathrm{O}$ (288,77): C, 66.55; H, 5.93; N, 9.70. Found, C, 66.28; H, 5.64; N, 9.48.

\section{(9aH)-6-Methylcyclopropa[9,10]ergoline-8-one hydrochloride (11)}

Yield: $6 \%, \mathrm{Mp}$ : decomposes over $200{ }^{\circ} \mathrm{C}$

${ }^{1} \mathrm{H}$ NMR (400 MHz, DMSO-d6) $\delta$ (ppm): 1.34 (1H, dd, $J=10.3,6.2 \mathrm{~Hz}, \mathrm{H} 9 \mathrm{y}$ ), 3.04 (1H, dd, J = 6.2, 5.2 Hz, H9 $\left.{ }_{\mathrm{x}}\right), 3.16(1 \mathrm{H}, \mathrm{dd}, J=10.3,5.2 \mathrm{~Hz}, \mathrm{H} 9 \mathrm{a}), 2.93\left(1 \mathrm{H}, \mathrm{m}, \mathrm{H} 4_{\mathrm{y}}\right), 2.94\left(3 \mathrm{H}, \mathrm{s}, \mathrm{N}^{+}-\mathrm{CH}_{3}\right), 3.22(1 \mathrm{H}, \mathrm{m}, \mathrm{H} 7 \mathrm{y}), 3.52$ $(1 \mathrm{H}, \mathrm{dd}, J=12.4,6.3 \mathrm{~Hz}, \mathrm{H} 7 \mathrm{x}), 3.64\left(1 \mathrm{H}, \mathrm{dd}, J=14.2,3.5 \mathrm{~Hz}, \mathrm{H} 4_{\mathrm{x}}\right), 3.88(1 \mathrm{H}, \mathrm{m}, \mathrm{H} 5), 6.53(1 \mathrm{H}, \mathrm{d}, J=7.2$ Hz, H12), 7.01 (1H, dd, $J=7.8,7.2 \mathrm{~Hz}, \mathrm{H} 13), 7.13$ (1H, s, H2), 7.17 (1H, d, J = 7.8 Hz, H14), 10.9 (1H, s, H1), $11.1\left(1 \mathrm{H}\right.$, br.s $\left.\mathrm{N}^{+}-\mathrm{H}\right)$.

${ }^{13} \mathrm{C}$ NMR (100 MHz, DMSO-d6) $\delta$ (ppm): 20.9 (C9), 23.8 (C4), 29.3 (C9a), 29.6 (C10), $41.8\left(\mathrm{~N}^{+}-\mathrm{CH}_{3}\right)$, 58.9 (C7), 60.8 (C5), 106.7 (C4), 109.7 (C12), 109.9 (C14), 120.0 (C2), 122.5 (C13), 126.2 (C15b), 129.0 (C11), 133.0 (C15a), 197.6 (C8).

Anal. Calcd. for $\mathrm{C}_{16} \mathrm{H}_{17} \mathrm{ClN}_{2} \mathrm{O}$ (288,77): C, 66.55; H, 5.93; N, 9.70. Found, C, 66.72; H, 5.88; N, 9.82. 


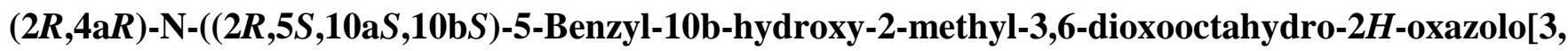
2-a]pyrrolo[2,1-c]pyrazin-2-yl)-4-methyl-1,1a,2,3,4,4a,5,7-octahydrocyclopropa[d]indolo[4,3-fg]qui noline-2-carboxamide hydrochloride (13)

Yield: $12 \%$, Mp: $196-206{ }^{\circ} \mathrm{C}$

${ }^{1} \mathrm{H}$ NMR (400 MHz, DMSO-d6) $\delta$ (ppm): 0.68 (1H, dd, $\left.J=9.9,5.6 \mathrm{~Hz}\right), 1.53(3 \mathrm{H}, \mathrm{s}), 1.72(1 \mathrm{H}, \mathrm{m}), 1.85$ $(1 \mathrm{H}, \mathrm{m}), 1.90(1 \mathrm{H}, \mathrm{m}), 1.97(1 \mathrm{H}, \mathrm{m}), 2.05(1 \mathrm{H}, \mathrm{t}, J=5.6 \mathrm{~Hz}), 2.24(1 \mathrm{H}, \mathrm{ddd}, J=9.9,5.6,1.5 \mathrm{~Hz}), 2.93(3 \mathrm{H}$, s), $2.97(1 \mathrm{H}, \mathrm{t}, \mathrm{J}=13.5 \mathrm{~Hz}), 3.12(1 \mathrm{H}, \mathrm{dd}, \mathrm{J}=14.2,4.4 \mathrm{~Hz}), 3.15-3.25(2 \mathrm{H}, \mathrm{m}), 3.30-3.50(5 \mathrm{H}, \mathrm{m}), 3.64$ $(1 \mathrm{H}, \mathrm{dd}, J=13.5,3.6 \mathrm{~Hz}), 3.90(1 \mathrm{H}, \mathrm{m}), 4.52(1 \mathrm{H}, \mathrm{t}, J=5.2 \mathrm{~Hz}), 6.42(1 \mathrm{H}, \mathrm{br} . \mathrm{s}), 6.47(1 \mathrm{H}, \mathrm{d}, J=7.6 \mathrm{~Hz})$, $7.00(1 \mathrm{H}, \mathrm{t}, J=7.6 \mathrm{~Hz}), 7.08-7.22(5 \mathrm{H}, \mathrm{m}), 7.32(2 \mathrm{H}, \mathrm{m}), 9.9(1 \mathrm{H}, \mathrm{s}), 10.9$ (1H, s), 11.3 (1H, br.s).

${ }^{13} \mathrm{C}$ NMR (100 MHz, DMSO-d6) $\delta$ (ppm): 15.8, 20.3, 21.7, 23.7, 24.1, 24.9, 25.9, 38.3, 41.7, 45.8, 53.5, $56.4,62.8,63.8,66.3,86.1,102.9,107.7,108.5,109.4,119.7,122.3,126.0,126.2,127.7$ (2C), 129.8 (2C), 132.7, 133.0, 138.7, 164.3, 165.9, 173.0 .

Anal.Calcd. for $\mathrm{C}_{34} \mathrm{H}_{37} \mathrm{~N}_{5} \mathrm{O}_{5}(595,69)$ : C, 68.55; H, 6.26; N, 11.76. Found, C, 68.80; H, 6.41; N, 11.50 .

\section{ACKNOWLEDGEMENTS}

The authors thank the National Fund for Science and Research (OTKA Project. No. T75705) for financial support.

\section{REFERENCES}

1. M. Incze, G. Dörnyei, I. Moldvai, E. Temesvári-Major, O. Egyed, Cs. Szántay, Tetrahedron, 2008, 64, 2924.

2. F. J. Weigert and J. D. Roberts, J. Am. Chem. Soc., 1967, 89, 5962.

3. K. B. Wiberg, Acc. Chem. Res., 1996, 29, 229.

4. H. Lebel, J.-F. Marcoux, C. Molinaro, and A. B. Charette, Chem. Rev., 2003, 103, 977.

5. R. Faust, Angew. Chem. Int. Ed., 2001, 40, 2251.

6. L. A. Wessjohann and W. Brandt, Chem. Rev., 2003, 103, 1625.

7. K. Yoshida, K. Yamaguchi, A. Mizuno, Y. Unno, A. Asai, T. Sone, H. Yokosawa, A. Matsuda, M. Arisawa, and S. Shuto, Org. Biomol.Chem., 2009, 7, 1868.

8. M. Tori, T. Hamaguchi, M. Aoki, M. Sono, and Y. Ashakawa, Can. J. Chem., 1997, 75, 634.

9. J. C. Lorenz, J. Long, Z. Yang, S. Yue, Y. Xie, and Y. Shi, J. Org. Chem., 2004, 69, 327.

10. I. Moldvai, E. Gács-Baitz, M. Balázs, M. Incze, and Cs. Szántay, Arch. Pharm. Pharm. Med. Chem., 1996, 329, 541.

11. R. M. Ortuno, J. Ibarzo, A. Alvarez-Larena, J. F. Piniella, Tetrahedron Lett., 1996 , 37, 4059.

12. W. A. Donaldson, Tetrahedron, 2001, 57, 8589. 
13. I. Moldvai, E. Gács-Baitz, E. Termesvári-Major, L. Russo, I. Pápai, K. Rissanen, É. Szárics, J. Kardos, and Cs. Szántay, Heterocycles, 2007, 71, 1075.

14. I. Moldvai, E. Temesvári-Major, M. Incze, É. Szentirmay, E. Gács-Baitz, and Cs. Szántay, J. Org. Chem., 2004, 69, 5993.

15. The MM2 calculation was carried out by ChemBio3D Ultra (CambridgeSoft) program. 